\title{
Coeficiente de heterogeneidade do solo e tamanho de parcela
}

\author{
Sueli Martins de Freitas Alves ${ }^{(1)}$ e José Carlos Seraphin ${ }^{(2)}$
}

(1)Universidade Estadual de Goiás, Unidade Universitária de Ciências Exatas e Tecnológicas, Caixa Postal 459, CEP 75110-390 Anápolis, GO. E-mail: suelifreitas@ueg.br (2)Universidade Federal de Goiás, Instituto de Matemática e Estatística, Caixa Postal 131, CEP 74001-970 Goiânia, GO. E-mail: seraphin@mat.ufg.br

\begin{abstract}
Resumo - O objetivo deste trabalho foi apresentar alternativas para estimar o coeficiente de heterogeneidade do solo a partir do coeficiente de correlação intraclasse em diferentes estruturas de delineamentos experimentais. As estimativas dos coeficientes de heterogeneidade do solo obtidas por este método foram comparadas àquelas obtidas por meio de mínimos quadrados generalizados. Não houve diferenças entre as estimativas obtidas por ambos os métodos; nesse caso recomenda-se a estimativa com base no coeficiente de correlação intraclasse por ser mais simples e prática. Considerando-se o coeficiente de heterogeneidade do solo, obteve-se diversos tamanhos de parcela, para diferentes combinações de coeficiente de variação, número de repetições, grau de diferença que se espera detectar entre dois tratamentos e nível de significância.

Termos para indexação: coeficiente de correlação intraclasse, precisão experimental, delineamento experimental, coeficiente de variação.
\end{abstract}

\section{Soil heterogeneity coefficient and plot size}

\begin{abstract}
The aim of this research was to present ways to estimate the coefficient of soil heterogeneity from the intraclass correlation coefficient for different structure of experimental designs. The coefficient of soil heterogeneity estimates obtained by this method were compared with those obtained by the generalized least square method. Practically, there was no difference between the estimates obtained from both methods, and thus it is recommended to use the estimate obtained from the coefficient of intraclass correlation due to its simplicity and practicability. Several plot sizes were obtained from the coefficient of soil heterogeneity estimates, for different combinations of variation coefficient, replicate number, expected difference between treatments and significance level.
\end{abstract}

Index terms: intraclass correlation coefficient, experimental precision, experimental design, variation coefficient.

\section{Introdução}

Em geral, o tamanho de parcelas é adotado tomandose por base apenas a literatura que apresenta propostas de parcelas que levam em consideração peculiaridades da região onde o experimento é conduzido. A literatura pode orientar teoricamente sobre qual tamanho se empregar, mas alguns critérios devem ser avaliados pelo pesquisador, tais como heterogeneidade do solo, natureza do material experimental, número de tratamentos, número de repetições, competição intercultivares, questões práticas e custo do experimento (Storck, 1979; Chaves, 1985; Bertolucci, 1990).

A espécie cultivada também é um fator de grande importância ao se determinar o tamanho de parcela, porque as diferentes culturas possuem necessidades fisiológicas próprias e por isso respondem diferentemente às variações ambientais (Bertolucci, 1990). O tamanho de parcela é uma característica particular de cada experimento, e apesar de poder variar segundo diversos fatores, há autores que consideram a heterogeneidade do solo como um fator determinante no tamanho de parcela (Storck, 1979; Bertolucci, 1990). Esta heterogeneidade pode se expressar de maneira diferente de ano para ano em um mesmo local (Hallauer, 1964).

$\mathrm{O}$ tamanho da parcela experimental das diferentes culturas tem sido tema de discussão desde Smith (1938). Embora existam vários procedimentos para estimar o tamanho mais conveniente de parcela, Hatheway (1961) propôs uma fórmula genérica que depende principalmen- 
te do coeficiente de heterogeneidade do solo. A dificuldade de se obter este coeficiente tem limitado o emprego dessa fórmula, uma vez que a maioria dos métodos para estimar o coeficiente de heterogeneidade do solo é muito complexa. Entre eles citam-se dois métodos que podem ser empregados tanto nos ensaios de uniformidade quanto nos ensaios que envolvem vários tratamentos. No primeiro, o coeficiente de heterogeneidade do solo é obtido a partir do coeficiente de correlação intraclasse (Binns, 1982; Lin \& Binns, 1984), e no segundo, a partir das variâncias de parcelas em função dos seus respectivos tamanhos, pelos mínimos quadrados generalizados (Rossetti \& PimentelGomes, 1983). Ambos os métodos dependem da estrutura do delineamento empregado.

O objetivo deste trabalho foi apresentar alternativas para estimar o coeficiente de heterogeneidade do solo a partir do coeficiente de correlação intraclasse em diferentes estruturas de delineamentos experimentais.

\section{Correlação intraclasse para diferentes estru- turas experimentais}

A correlação intraclasse está intrinsecamente relacionada com a maneira pela qual a correlação é obtida e pode ser definida como a proporção da variância total associada com a classe à qual a observação pertence. Assim, se dois membros da mesma classe são correlacionados, a esta correlação dá-se o nome de correlação intraclasse. Uma classe pode ser definida como uma variedade, um bloco, uma parcela, um nível de hierarquia.

A estimativa do coeficiente de correlação intraclasse $(\hat{\rho})$ pode ser obtida a partir dos componentes de variância da análise de variância. A Tabela 1 apresenta o esquema de análise de variância para alguns delineamentos experimentais, bem como as esperanças de quadrados médios, considerando os tratamentos com efeito fixo. Neste caso, as estimativas dos componentes de variância foram obtidas igualando-se os quadrados médios da análise com suas respectivas esperanças matemáticas, e a estimativa do coeficiente de correlação intraclasse é obtida igualando-se as esperanças matemáticas com as respectivas esperanças matemáticas em que se consideram que exista correlação entre observações de mesma classe.

A Tabela 2 mostra as diferentes formas para obter o coeficiente de correlação intraclasse de acordo com o delineamento e o tipo de correlação intraclasse de interesse. Contudo, a expressão para obter este coeficien- te, considerando o ensaio hierárquico, foi obtida com base em Kemphtorne (1957). Segundo esse autor, podese mostrar a esperança matemática quando se considera que haja correlação entre subparcelas dentro do bloco, no caso do delineamento em blocos completos casualizados com parcelas subdivididas (Tabela 1).

\section{Obtenção do coeficiente de heterogeneidade do solo e tamanho de parcela}

Um estimador para o coeficiente de heterogeneidade do solo (b) é apresentado por Lin \& Binns (1984) para um delineamento em blocos completos casualizados com I parcelas por bloco e é definido como

$\hat{\mathrm{b}}=1-\frac{\log [\mathrm{m}-(\mathrm{m}-1)(1-\hat{\rho})]}{\log \mathrm{m}}$

em que m é o número I de parcelas por bloco; $\hat{\rho}=\frac{\sigma_{b}^{2}}{\sigma^{2}+\sigma_{b}^{2}}$ é a estimativa do coeficiente de correlação intraclasse.

A expressão (1) pode ser generalizada para outros delineamentos, redefinindo-se os valores de $\mathrm{m}$ e reexpressando os estimadores de $\rho$ (Tabela 2).

Uma vez obtido o coeficiente de heterogeneidade do solo, o tamanho de parcela pode ser estimado pela fórmula proposta por Hatheway (1961):

$\mathrm{x}^{\mathrm{b}}=\frac{2\left(\mathrm{t}_{1}+\mathrm{t}_{2}\right)^{2} \mathrm{C}^{2}}{\mathrm{rd}^{2}}$

em que x é o número de unidades básicas que compõem a parcela; $b$ é o coeficiente de heterogeneidade do solo; $\mathrm{t}_{1}$ é o valor crítico de $\mathrm{t}$ de Student para o nível de significância $\mathrm{a}_{1} ; \mathrm{t}_{2}$ é o valor tabelado de $\mathrm{t}$ de Student, correspondendo a $\mathrm{a}_{2}=2(1-\mathrm{p})$, em que $\mathrm{p}$ é a probabilidade de se obter diferenças significativas entre as médias; $\mathrm{C}$ é o coeficiente de variação (\%); r é o número de repetições; d é a diferença que se espera detectar entre dois tratamentos $(\%)$.

\section{Aplicação}

Os valores de $\rho$ e de $b$ foram obtidos considerandose três experimentos: dois ensaios com milho verde, desenvolvidos na Embrapa Arroze Feijão, Santo Antônio de Goiás, GO, e um ensaio de uniformidade com batata, apresentado por Cordeiro et al. (1982). O primeiro ensaio de milho verde foi instalado em maio de 1995 (época 1) e o segundo em agosto do mesmo ano (época 2), e a variável considerada na análise foi peso de espigas com palha. As parcelas experimentais foram compostas de 


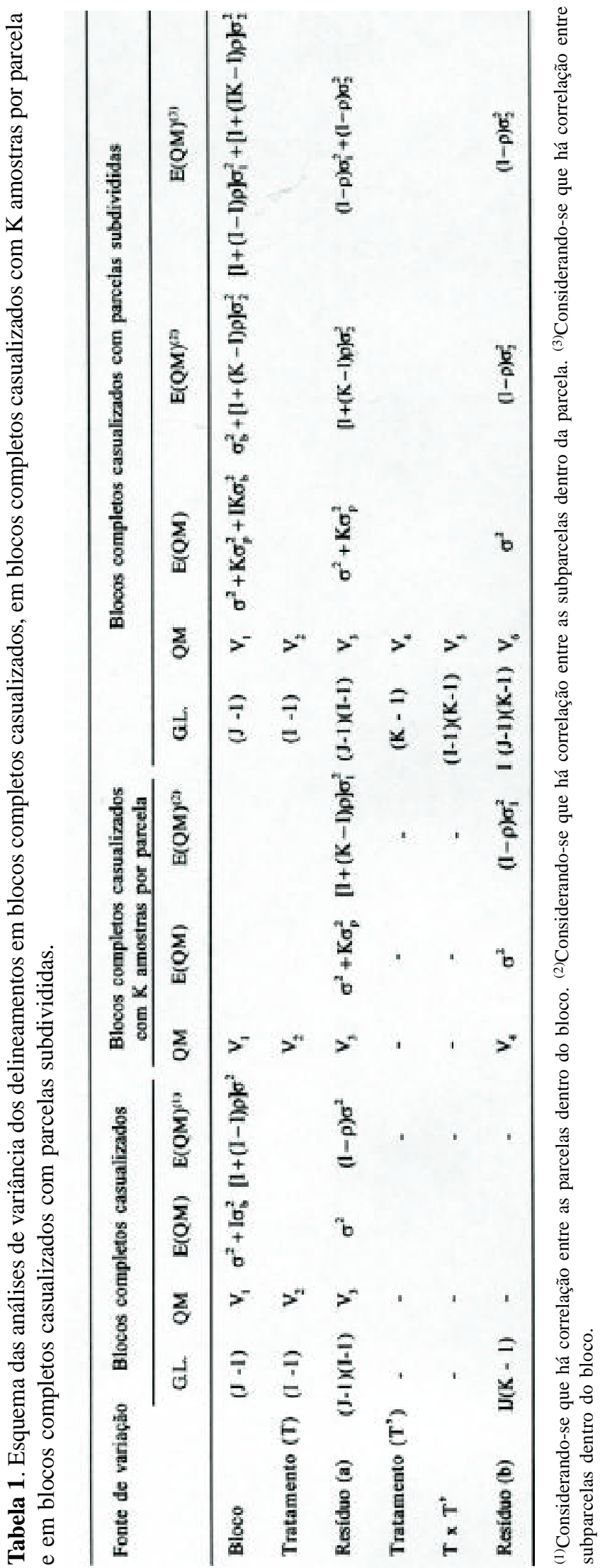

seis linhas de cinco metros de comprimento. Na análise do ensaio de batata, foi utilizado o resultado da análise de variância no esquema hierárquico, apresentado por Cordeiro et al. (1982). O valor de b foi calculado de acordo com as generalizações propostas na Tabela 2 para estes três experimentos usando a expressão (1) (método 1) e, para efeito de comparação, estas estimativas também foram obtidas a partir do método de mínimos quadrados generalizados (método 2), apresentado por Rossetti \& Pimentel-Gomes (1983) e Alves (1999), tendo ambos por base o método de Hatheway \& Williams (1958).

As estimativas de $\mathrm{b}$ obtidas a partir dos métodos 1 e 2 foram praticamente similares (Tabela 3 ). Resultado semelhante foi obtido por Lin \& Binns (1984), quando compararam a estimativa de $\hat{b}$ obtida por eles com a estimativa obtida por Koch \& Rigney (1951). Houve uma consistência nos resultados quando compararamse os dois métodos, e, além disso, o método que utiliza o coeficiente de correlação intraclasse é mais simples e prático.

Apesar de os ensaios com milho verde terem sido realizados no mesmo ano, os valores de b e $\rho$ encontrados devem ser considerados como aproximações, uma vez que podem variar de ano para ano, conforme Lin \& Binns (1984). Para verificar se as estimativas são consistentes de ano para ano, deveriam ser realizados plantios destas culturas por anos consecutivos nas áreas consideradas. Estudo desenvolvido por Hallauer (1964), em experimentos de milho para grãos, em plantios realizados em 15 locais durante nove anos, houve variação considerável de ano para ano em alguns locais, enquanto em outros praticamente não houve variação nas estimativas obtidas.

Na Tabela 4, são apresentados os vários tamanhos de parcela para milho verde, obtidos pela fórmula Hatheway (1961), nas diferentes combinações de coeficiente de heterogeneidade do solo, coeficiente de variação, diferenças que se espera detectar entre médias de dois tratamentos e número de repetições, considerando um nível de significância de $5 \%\left(\alpha_{1}=0,05\right)$ e uma probabilidade de se obter diferenças significativas entre as médias de $80 \%(\mathrm{p}=0,80)$.

As estimativas de tamanho de parcela são fornecidas em número de linhas úteis, e os valores obtidos foram arredondados para o inteiro superior. Fixando-se o grau de precisão d, por exemplo, em 20\% (Tabela 4), o tamanho de parcela será de 107 linhas, considerando-se duas repetições, e de nove linhas, considerando-se cin- 
co repetições, para $b=0,4$ e um CV de $15 \%$. O aumento no número de repetições parece ser mais eficiente do que o aumento no tamanho da parcela, no sentido de melhor aproveitamento da área experimental. Além disso, parcelas experimentais excessivamente grandes tornam o trabalho mais difícil e oneroso (Storck, 1979; Vieira, 1996). Portanto, fixando-se o valor de d e reduzindo-se o tamanho de parcela, um maior número de repetições será exigido e, conseqüentemente, um aumento no tamanho da parcela fará com que um menor número de repetições seja necessário.

Estes resultados revelam que existe uma relação inversa entre o tamanho de parcela e o número de repetições, ou seja, quanto maior o número de repetições uti- lizados menor deverá ser o tamanho da parcela experimental, o que está de acordo com as observações de Hatheway (1961), Ramalho et al. (1977) e Resende \& Souza Júnior (1997). Quando comparam-se parcelas com o mesmo número de linhas, verifica-se que, para um número maior de repetições, pode-se detectar uma menor diferença mínima entre médias de tratamento. Considerando-se uma parcela de duas linhas úteis, observa-se que, com quatro repetições, pode-se detectar uma diferença mínima de $20 \%$ entre médias de tratamentos, ao passo que, considerando apenas três repetições, o valor da diferença mínima passa a ser de $25 \%$, para um CV de $10 \%$ e $b=0,4$. O aumento no número de repetições parece ser mais eficiente na detecção de

Tabela 2. Estimadores do coeficiente de correlação intraclasse, de acordo com o delineamento experimental e o tipo de correlação intraclasse ${ }^{(1)}$.

\begin{tabular}{|c|c|c|c|}
\hline Delineamento & Tipo de correlação intraclasse & Estimador de $\rho$ & $\begin{array}{l}\text { Número de unidades } \\
\text { por classe }(\mathrm{m})\end{array}$ \\
\hline $\begin{array}{l}\text { Blocos completos casualizados com } \mathrm{K} \\
\text { amostras por parcela }\end{array}$ & Entre amostras dentro da parcela & $\frac{\sigma_{p}^{2}}{\sigma^{2}+\sigma_{p}^{2}}$ & K \\
\hline $\begin{array}{l}\text { Blocos completos casualizados com } \\
\text { parcelas subdivididas }\end{array}$ & Entre subparcelas dentro da parcela & $\frac{\sigma_{p}^{2}}{\sigma^{2}+\sigma_{p}^{2}}$ & $\mathrm{~K}$ \\
\hline $\begin{array}{l}\text { Blocos completos casualizados com } \\
\text { parcelas subdivididas }\end{array}$ & Entre subparcelas dentro do bloco & $\frac{\sigma_{\mathrm{b}}^{2}}{\sigma^{2}+\sigma_{\mathrm{p}}^{2}+\sigma_{\mathrm{b}}^{2}}$ & IK \\
\hline $\begin{array}{l}\text { Hierárquico com cinco níveis de } \\
\text { hierarquia (ABCDE), com parcelas do } \\
\text { tipo } \varepsilon \text {, dentro de parcelas do tipo } \delta \text {, } \\
\text { dentro de parcelas do tipo } \lambda \text {, dentro de } \\
\text { parcelas do tipo } \beta \text {, dentro de parcelas } \\
\text { do tipo } \alpha \text {. }\end{array}$ & $\begin{array}{l}\text { Entre parcelas do tipo } \varepsilon \text {, dentro de } \\
\text { parcelas do tipo } \delta \text {, dentro de parcelas } \\
\text { do tipo } \lambda \text {, dentro de parcelas do tipo } \beta \text {, } \\
\text { dentro de parcelas do tipo } \alpha \text {. }\end{array}$ & $\frac{\sigma_{\alpha}^{2}}{\sigma_{\alpha}^{2}+\sigma_{\beta}^{2}+\sigma_{\lambda}^{2}+\sigma_{\delta}^{2}+\sigma_{\varepsilon}^{2}}$ & $\mathrm{BCDE}$ \\
\hline
\end{tabular}

${ }^{(1)} \mathrm{K}$ é o número de amostras ou subparcelas dentro da parcela; A é o número de parcelas do tipo $\alpha$; B é o número de parcelas do tipo $\beta$; C é o número de parcelas do tipo $\lambda$; D é o número de parcelas do tipo $\delta$; E é o número de parcelas do tipo $\varepsilon$.

Tabela 3. Estimativa do coeficiente de heterogeneidade do solo $(\hat{b})$, a partir das estimativas do coeficiente de correção intraclasse ( $\hat{\rho}$ ) (método 1) e pelo método de mínimos quadrado generalizados (método 2).

\begin{tabular}{|c|c|c|c|c|c|c|c|c|c|}
\hline \multirow[t]{3}{*}{ Ensaios } & \multicolumn{3}{|c|}{$\begin{array}{l}\text { Delineamento em blocos } \\
\text { completos casualizados }\end{array}$} & \multicolumn{3}{|c|}{$\begin{array}{c}\text { Delineamento em blocos completos } \\
\text { casualizados com parcelas subdivididas }^{(1)}\end{array}$} & \multicolumn{3}{|c|}{$\begin{array}{c}\text { Delineamento } \\
\text { hierárquico }\end{array}$} \\
\hline & \multicolumn{2}{|c|}{ Método 1} & \multirow{2}{*}{$\frac{\text { Método } 2}{\hat{\mathrm{b}}}$} & \multicolumn{2}{|c|}{ Método 1} & \multirow{2}{*}{$\frac{\text { Método } 2}{\hat{\mathrm{b}}}$} & \multicolumn{2}{|c|}{ Método 1} & \multirow{2}{*}{$\begin{array}{c}\text { Método } 2 \\
\hat{\mathrm{b}}\end{array}$} \\
\hline & $\hat{\rho}$ & $\hat{b}$ & & $\hat{\rho}$ & $\hat{b}$ & & $\hat{\rho}$ & $\hat{b}$ & \\
\hline Milho verde (época 1) & 0,23 & 0,40 & 0,40 & 0,19 & 0,40 & 0,30 & - & - & - \\
\hline Milho verde (época 2) & 0,15 & 0,50 & 0,50 & 0,07 & 0,60 & 0,60 & - & - & - \\
\hline Batata & - & - & - & - & - & - & 0,0022 & 0,92 & $0,93^{(2)}$ \\
\hline
\end{tabular}

${ }^{(1)}$ Considerando o coeficiente de correlação intraclasse entre subparcelas dentro do bloco. (2) Resultado obtido por Cordeiro et al. (1982). 
uma porcentagem menor de diferenças entre médias. Essa relação entre número de repetições e a diferença mínima entre médias de tratamentos pode ser observada também em Pimentel-Gomes (1990).

Há uma influência acentuada do coeficiente de variação sobre o tamanho da parcela, pois quanto maior $o$ $\mathrm{CV}$, mais acentuada a alteração no tamanho da parcela com a mudança da heterogeneidade do solo. Diversos autores têm mostrado a existência de uma relação inversa entre o tamanho da parcela e o erro experimental e, dessa forma, o aumento no tamanho da parcela leva a um decréscimo no coeficiente de variação (Igue \& Mascarenhas, 1974; Ramalho et al., 1977; Zimmermann, 1982; Resende \& Souza Júnior, 1997). Essa relação ocorre quando avaliam-se apenas esses dois fatores isoladamente, ou seja, tamanho de parcela e coeficiente de variação. Entretanto, essa relação não é tão simples como se apresenta, pois deve-se considerar que fatores tais como a heterogeneidade do solo e número de repetições também a influenciam (Tabela 4). Observa-se, por exemplo, que há situações em que quanto maior o $\mathrm{CV}$ maior o tamanho da parcela, principalmente quando considera-se menor $\mathrm{r}$ e menor $\mathrm{d}$.

Com relação ao tamanho de parcela e coeficiente de heterogeneidade do solo b, de uma maneira geral, verifica-se que, para um CV fixo, ao aumentar o coeficiente de heterogeneidade do solo, o tamanho da parcela diminui e essa alteração no tamanho da parcela é mais acentuada na medida em que o número de repetições $r$ é menor, assim como a diferença que se deseja detectar d. A princípio, estes resultados parecem contraditórios em relação aos trabalhos apresentados com maior freqüência na literatura, pois vários deles sugerem a utilização de parcelas maiores em áreas experimentais heterogêneas e parcelas menores em áreas mais homogêneas (Igue \& Mascarenhas, 1974; Storck, 1979; Resende \& Souza Júnior, 1997). Contudo, essa relação considera apenas a heterogeneidade do solo como o fator determinante na obtenção da estimativa do tamanho da parcela. Pode haver diferenças entre os resultados obtidos por meio da utilização de diferentes métodos, conforme observado por Storck (1979), porém ainda há pouca discussão sobre esse tema.

Nos trabalhos de Igue \& Mascarenhas (1974) e Ramalho et al. (1977), os quais também utilizaram a fórmula de Hatheway (1961), os resultados foram semelhantes aos deste trabalho. Ao estimar tamanho de parcela pelo método de Hatheway (1961), não é apenas a heterogeneidade do solo que está sendo levada em con- 
sideração. Fatores como o delineamento experimental a ser usado, o número de tratamentos, o coeficiente de variação, a diferença que se espera detectar entre tratamentos e o número de repetições também estão envolvidos na estimativa do tamanho da parcela e a relação entre todos esses fatores é que será determinante no tamanho da parcela. A quantidade de informações fornecida por este método foi discutida por Oliveira (1976), quando realizou uma comparação entre alguns métodos para estimar tamanho de parcela.

$\mathrm{Na}$ expressão (2), denominando-se o termo por $\mathrm{H}$, $\frac{2\left(\mathrm{t}_{1}+\mathrm{t}_{2}\right)^{2} \mathrm{C}^{2}}{\mathrm{rd}^{2}}$ pode-se estabelecer duas situações. A primeira, se o $\mathrm{H}$ for maior do que um, ao aumentar o valor de $b$, diminui-se o tamanho da parcela, e a segunda, se o $\mathrm{H}$ estiver entre zero e um, ao aumentar o valor de $b$, aumenta-se o tamanho da parcela. Verifica-se que o coeficiente de variação continua apresentando uma acentuada influência na determinação do tamanho da parcela.

Quanto à utilização do coeficiente de correlação intraclasse $(\rho)$ para determinar tamanho de parcela, deve-se levar em consideração que, entre as regras práticas propostas por Lin \& Binns (1984), tem-se que, quando $\rho$ está na faixa $0,1 \leq \rho \leq 0,5$, a precisão experimental melhora, aumentando o tamanho da parcela e reduzindo o tamanho do bloco. Contudo, essa regra é muito geral, restando dúvidas de qual deve ser o tamanho da parcela, e o número de repetições. Somente essas regras não respondem a tais dúvidas. Outro exemplo de utilização do coeficiente de correlação intraclasse para estimar tamanho de parcela é visto em Alves et al. (2000) e, apesar de ser um método prático, também fornece informações limitadas. $\mathrm{O}$ valor do coeficiente de correlação intraclasse por eles determinado, em relação ao delineamento em blocos completos casualizados com $\mathrm{K}$ amostras por parcela, pelo estimador apresentado na Tabela 2, pode ser utilizado para obter o coeficiente de heterogeneidade do solo, conforme generalização da expressão (1) e, posteriormente aplicado na fórmula de Hatheway (1961).

\section{Conclusão}

A estimativa do coeficiente de heterogeneidade do solo obtida a partir do coeficiente de correlação intraclasse é um método simples e prático.

\section{Referências}

ALVES, S.M.F. Bordadura lateral e tamanho de parcela para ensaios de avaliação de genótipos de milho verde. 1999. 94p. Dissertação (Mestrado) - Universidade Federal de Goiás, Goiânia.

ALVES, S.M.F.; SERAPHIN, J.C.; ZIMMERMANN, F.J.P.; SILVA, A.E. Tamanho de parcela para ensaios de milho verde. Revista de Agricultura, v.75, p.235-246, 2000.

BERTOLUCCI, F.L.G. Novas alternativas de tamanho e forma da parcela experimental para avaliação de progênies do feijoeiro. 1990. 150p. Dissertação (Mestrado) - Escola Superior de Agricultura de Lavras, Lavras.

BINNS, M.R. The choice of plot size in randomised block experiments. Journal of the American Society for Horticultural Science, v.107, p.17-19, 1982.

CHAVES,L.J. Tamanho de parcela para seleção de progênies de milho (Zea mays L.). 1985. 148p. Tese (Doutorado) - Escola Superior de Agricultura Luiz de Queiroz, Piracicaba.

CORDEIRO, C.M.T.; MIRANDA, J.E.C.; CAMPOS, J. Tamanho de parcelas e número de repetições em experimento de batata. Pesquisa Agropecuária Brasileira, v.17, p.1341-1348, 1982.

HALLAUER, A.R. Estimation of soil variability and convenient plot size from corn trials. Agronomy Journal, v.56, p.493-497, 1964.

HATHEWAY, W.H. Convenient plot size. Agronomy Journal, Madison, v.53, p.279-280, 1961.

HATHEWAY, W.H.; WILLIAMS, E.J. Efficient estimation of the relationship between plot size and the variability of crop yields. Biometrics, v.14, p.207-222, 1958.

IGUE, T.; MASCARENHAS, H.A.A. Tamanho das parcelas para experimentos de campo com soja. Campinas: Instituto Agronômico, 1974. 28p. (Boletim Técnico, 9).

KEMPTHORNE, O. An introduction to genetic statistics, NewYork: J.Wiley, 1957. 545p.

KOCH, E.J.; RIGNEY, J.A.A. Method of estimating optimum plot size from experimental data. Agronomy Journal, v.43, p.17-21, 1951.

LIN, C.S.; BINNS, M.R. Working rules for determining the plot size and numbers of plots per block in field experiments. Journal of Agricultural Science, v.103, p.11-15, 1984.

OLIVEIRA, R.P. Estudo comparativo de alguns métodos de estimação do tamanho adequado de parcelas experimentais. 1976. 110p. Dissertação (Mestrado) - Universidade de Brasília, Brasília.

PIMENTEL-GOMES, F. Curso de Estatística experimental. 13.ed. Piracicaba: USP, 1990. 467p.

RAMALHO, M.A.P.; DUARTE, G.S.; SILVEIRA, J.V.; CARVALHO, M.A. Estimativa do tamanho da parcela para os experimentos com a cultura do feijão. Ciência e Prática, v.1, p.5$12,1977$. 
RESENDE, M.D.V.; SOUZA JÚNIOR, C.L. Número de repetições e tamanho de parcela para seleção de progênies de milho em solos sob cerrado e fértil. Pesquisa Agropecuária Brasileira, v.32, p.781$788,1997$.

ROSSETTI, A.G.; PIMENTEL-GOMES, F. Determinação do tamanho ótimo de parcelas em ensaios agrícolas. Pesquisa Agropecuária Brasileira, v.18, p.444-487, 1983.

SMITH, H.F. An empirical law describing heterogeneity in the yields of agricultural crops. Journal of Agricultural Science, v.28, p.123,1938
STORCK,L. Estimativa do tamanho e forma de parcela e número de repetições para experimentos com milho (Zea mays L.). 1979. 90p. Dissertação (Mestrado) - Universidade Federal do Rio Grande do Sul, Porto Alegre.

VIEIRA, N.E. Tamanho e forma de parcela experimental para avaliação de genótipos de arroz (Oryza sativa L.) de sequeiro. 1996. 98p. Dissertação (Mestrado) - Universidade Federal de Goiás, Goiânia.

ZIMMERMANN, F.J.P. Tamanho de parcela para pesquisa de feijão consorciado com milho. Pesquisa Agropecuária Brasileira, v.17, p.741-743, 1982.

Recebido em 9 de junho de 2003 e aprovado em 2 de dezembro de 2003 
